\title{
On the Existence of Solutions for Impulsive Fractional Differential Equations
}

\author{
Yongliang Guan, Zengqin Zhao, and Xiuli Lin \\ School of Mathematical Sciences, Qufu Normal University, Qufu 273165, China \\ Correspondence should be addressed to Zengqin Zhao; zqzhaoy@163.com
}

Received 3 July 2017; Accepted 16 October 2017; Published 7 December 2017

Academic Editor: André Nicolet

Copyright (c) 2017 Yongliang Guan et al. This is an open access article distributed under the Creative Commons Attribution License, which permits unrestricted use, distribution, and reproduction in any medium, provided the original work is properly cited.

\begin{abstract}
We are concerned with a type of impulsive fractional differential equations attached with integral boundary conditions and get the existence of at least one positive solution via global bifurcation techniques.
\end{abstract}

\section{Introduction}

Fractional differential equations have been extensively studied in recent years (see, for instance, [1-7] and their references). In addition, since Rabinowitz established unilateral global bifurcation theorems, there have been many researches in global bifurcation theory and it has been applied to obtain the existence and multiplicity for solutions of differential equations (see, for instance, [8-16] and their references). However, the previous researches seldom involve both global bifurcation techniques and fractional differential equations. In [16], the following problem was studied.

$$
\begin{aligned}
D_{0+}^{\alpha} u(t)+r f(t, u(t)) & =0, \quad t \in(0,1), \\
\left.t^{n-\alpha} u^{(n-2)}(t)\right|_{t=0} & =\left.t^{n-\alpha} u^{(n-3)}(t)\right|_{t=0} \cdots \\
& =\left.t^{n-\alpha} u(t)\right|_{t=0}=u(1)=0,
\end{aligned}
$$

where the fractional difference was of Riemann-Liouville type. Under suitable conditions, the existence of at least one positive solution and one negative solution was got. We would like to conduct further research on the above problem. For instance, in practical applications, $f$ may rely not only on $u(t)$ but also on $u^{\prime}(t)$, which will give rise to additional difficulties for the study. Moreover, what if the boundary value conditions are nonlocal rather than local? Can we add impulsive terms into the system? As a reply to above questions, we will tackle the following impulsive Caputo fractional differential equations attached with integral boundary value conditions in this paper.

$$
\begin{aligned}
{ }^{c} D_{0+}^{\alpha} u(t)+a f\left(t, u(t), u^{\prime}(t)\right) & =0, \quad t \in(0,1), \\
u^{\prime}(0) & =0, \\
u(1) & =\int_{0}^{1} k(t) u(t) d t, \\
\Delta u(\xi) & =\int_{0}^{\xi} g(t) u(t) d t, \\
\Delta u^{\prime}(\xi) & =\int_{0}^{\xi} h(t) u(t) d t,
\end{aligned}
$$

where $f \in C([0,1] \times \mathbb{R} \times \mathbb{R}, \mathbb{R})$ satisfies $f(t, x, y) \geq 0$ when $x \geq 0$ and $y \leq 0 ; k \in C([0,1],[0,+\infty]) ; \xi \in(0,1) ; g, h \in$ $C([0, \xi],(-\infty, 0])$;

$$
\begin{gathered}
\Delta u(\xi):=\lim _{t \rightarrow \xi_{+}} u(t)-\lim _{t \rightarrow \xi_{-}} u(t) \\
\Delta u^{\prime}(\xi):=\lim _{t \rightarrow \xi_{+}} u^{\prime}(t)-\lim _{t \rightarrow \xi_{-}} u^{\prime}(t) .
\end{gathered}
$$

We set $1<\alpha \leq 2$ throughout this paper.

Through global bifurcation techniques, we get the existence of at least one positive solution of (2) (see Theorem 14). Moreover, for the sake of convenience of use, we give a corollary of Theorem 14 (see Theorem 15). 
The rest of this paper is organized as follows. In Section 2, we will present some preliminary knowledge and some conditions for (2) that we need. We will prove some properties of several functions in Section 3 as a preparation of Section 4. In Section 4, we will present our main results and prove them. In Section 5, we present an example as an application of the main results. Finally, an appendix is given to prove a formula which will be used in the proof of the main results.

\section{Preliminary}

Firstly, we introduce several spaces that this paper needs.

In this paper, we set

$$
P C[0,1]=\{g: g(t) \text { are continuous on }[0, \xi],(\xi, 1] \text {; }
$$

there exists $\left.\lim _{t \rightarrow \xi_{+}} g(t)\right\}$

as well as

$$
P C^{1}[0,1]=\left\{g: g, g^{\prime} \in P C[0,1]\right\} .
$$

Secondly, we introduce some knowledge of fractional integral and fractional derivative.

Definition 1 (see [3]). The fractional integral of order $\beta>0$ for a function $g$ is defined as

$$
I_{0}^{\beta} g(t)=\frac{1}{\Gamma(\beta)} \int_{0}^{t}(t-s)^{\beta-1} g(s) d s, \quad t \geq 0
$$

Definition 2 (see [3]). The Riemann-Liouville derivative of fractional order $\alpha$ for a function $g$ is defined as

$$
{ }^{r l} D_{0}^{\alpha} g(t)=\frac{d^{2}}{d t^{2}} I_{0}^{2-\alpha} g(t), \quad t>0 .
$$

For $g \in L_{p}(a, b)(1 \leq p \leq+\infty)$, it is well known that ${ }^{r l} D_{0}^{\alpha} I_{0}^{\alpha} g(t)=g(t)$ holds almost everywhere on $[a, b]$. What is more, ${ }^{r l} D_{0}^{\alpha} I_{0}^{\alpha} g(t)=g(t)$ holds on $(a, b)$ if $g \in C(a, b)$.

Definition 3 (see [3]). The Caputo derivative of fractional order $\alpha$ for a function is defined as

$$
{ }^{c} D_{0}^{\alpha} g(t)={ }^{r l} D_{0}^{\alpha}\left[g(t)-g(0)-g^{\prime}(0) t\right], \quad t \in[0,1] .
$$

Clearly, (8) is equivalent to

$$
\begin{array}{r}
{ }^{c} D_{0}^{\alpha} g(t)={ }^{r l} D_{0}^{\alpha} g(t)-\frac{g(0)}{\Gamma(1-\alpha)} t^{-\alpha}-\frac{g^{\prime}(0)}{\Gamma(2-\alpha)} t^{1-\alpha}, \\
t \in[0,1] .
\end{array}
$$

Moreover, for $g \in C^{1}[0,1]$ and $\phi \in L[0,1]$, it is well known that

$$
{ }^{c} D_{0}^{\alpha} g(t)=\phi(t) \Longrightarrow g(t)=I_{0}^{\alpha} \phi(t)+C_{0}+C_{1} t .
$$

However, (10) does not necessarily hold if $g$ does not belong to $C^{1}[0,1]$. Since $u(t)$ and $u^{\prime}(t)$ considered in (2) are not continuous at $t=\xi$, we need some modification on Definition 3. So we generalize Definition 3 in the following way, which will be applied to (2).

Definition 4. The Caputo derivative of fractional order $\alpha$ for a function $g \in P C^{1}[0,1]$ is defined as

$$
{ }^{c} D_{0}^{\alpha} g(t)={ }^{r l} D_{0}^{\alpha}\left(g-g_{\xi}\right)(t), \quad t \in[0,1],
$$

where

$$
g_{\xi}(t):= \begin{cases}g(0)+g^{\prime}(0) t, & t \in[0, \xi], \\ g(0)+g\left(\xi_{+}\right)-g\left(\xi_{-}\right)+g^{\prime}(0) t+g^{\prime}\left(\xi_{+}\right)(t-\xi)-g^{\prime}\left(\xi_{-}\right)(t-\xi), & t \in(\xi, 1] .\end{cases}
$$

With the above definition, we have a conclusion similar to (10), which will be represented as (13).

Lemma 5. For $g \in P C^{1}[0,1]$ and $\phi \in L[0,1]$, if ${ }^{c} D_{0}^{\alpha} g(t)=$ $\phi(t)$ for $t \in(0,1)$, there must be

$$
g(t)= \begin{cases}I_{0}^{\alpha} \phi(t)+C_{1}+C_{2} t, & t \in[0, \xi) \\ I_{0}^{\alpha} \phi(t)+C_{3}+C_{4} t, & t \in(\xi, 1]\end{cases}
$$

Proof. Suppose that

that is

$$
{ }^{c} D_{0}^{\alpha} g(t)=\phi(t), \quad t \in(0,1) ;
$$

$$
{ }^{r l} D_{0}^{\alpha}\left(g-g_{\xi}\right)(t)=\phi(t), \quad t \in(0,1) .
$$

We know that

$$
g(t)-g_{\xi}(t)= \begin{cases}I_{0}^{\alpha} \phi(t)+d_{1} t^{\alpha-1}+d_{2} t^{\alpha-2}, & t \in[0, \xi), \\ I_{0}^{\alpha} \phi(t)+d_{1} t^{\alpha-1}+d_{2} t^{\alpha-2}+d_{3}(t-\xi)^{\alpha-1}+d_{4}(t-\xi)^{\alpha-2}, & t \in(\xi, 1]\end{cases}
$$


Then

$$
g^{\prime}(t)-g_{\xi}^{\prime}(t)= \begin{cases}I_{0}^{\alpha-1} \phi(t)+d_{1}(\alpha-1) t^{\alpha-2}+d_{2}(\alpha-2) t^{\alpha-3}, & t \in[0, \xi), \\ I_{0}^{\alpha-1} \phi(t)+d_{1}(\alpha-1) t^{\alpha-2}+d_{2}(\alpha-2) t^{\alpha-3}+d_{3}(\alpha-1)(t-\xi)^{\alpha-2}+d_{4}(\alpha-2)(t-\xi)^{\alpha-3}, & t \in(\xi, 1] .\end{cases}
$$

Since $g, g_{\xi} \in P C^{1}[0,1]$, we know that $g-g_{\xi} \in P C^{1}[0,1]$. Then by (17) we know that

$$
d_{1}=d_{2}=d_{3}=d_{4}=0 \text {. }
$$

So (13) turns to be

$$
g(t)-g_{\xi}(t)=I_{0}^{\alpha} \phi(t), \quad t \in[0,1] ;
$$

that is

$$
g(t)=I_{0}^{\alpha} \phi(t)+g_{\xi}(t), \quad t \in[0,1] .
$$

Choosing suitable $C_{1}, C_{2}, C_{3}, C_{4}$, we finish the proof.

If we set

$$
C(t)= \begin{cases}a+b t, & t \in[0, \xi] \\ c+d t, & t \in(\xi, 1]\end{cases}
$$

where $a, b, c$, and $d$ are constant real numbers, then we will have the following conclusion.

Lemma 6. One has

$$
{ }^{c} D_{0}^{\alpha} C(t)=0, \quad t \in[0,1] .
$$

Proof. It is easy to see that $C(t)-C_{\xi}(t)=0$ for $t \in[0,1]$ and then it is clear that

$$
{ }^{c} D_{0}^{\alpha} C(t)=0, \quad t \in[0,1] .
$$

Lemma 7. For $p(t) \in P C[0,1], u \in X$ is a solution of

$$
\begin{aligned}
{ }^{c} D_{0}^{\alpha} u(t)+p(t) & =0, \quad t \in(0,1), \\
u^{\prime}(0) & =0, \\
u(1) & =\int_{0}^{1} k(t) u(t) d t, \\
\Delta u(\xi) & =\int_{0}^{\xi} g(t) u(t) d t, \\
\Delta u^{\prime}(\xi) & =\int_{0}^{\xi} h(t) u(t) d t
\end{aligned}
$$

if and only if

$$
\begin{aligned}
u(t)= & -\frac{1}{\Gamma(\alpha)} \int_{0}^{t}(t-s)^{\alpha-1} p(s) d s \\
& +\frac{1}{\Gamma(\alpha)} \int_{0}^{1}(1-s)^{\alpha-1} p(s) d s \\
& -\int_{0}^{\xi} g(s) u(s) d s
\end{aligned}
$$

$$
-(1-\xi) \int_{0}^{\xi} h(s) u(s) d s
$$$$
+\int_{0}^{1} k(s) u(s) d s, \quad t \in[0, \xi]
$$

$$
\begin{aligned}
u(t)= & -\frac{1}{\Gamma(\alpha)} \int_{0}^{t}(t-s)^{\alpha-1} p(s) d s \\
& +\frac{1}{\Gamma(\alpha)} \int_{0}^{1}(1-s)^{\alpha-1} p(s) d s \\
& -(1-t) \int_{0}^{\xi} h(s) u(s) d s \\
& +\int_{0}^{1} k(s) u(s) d s, \quad t \in(\xi, 1] .
\end{aligned}
$$

Proof. If $u \in X$ is a solution of (24), by Lemma 5 we know that

$$
\begin{aligned}
& u(t) \\
& = \begin{cases}-\frac{1}{\Gamma(\alpha)} \int_{0}^{t}(t-s)^{\alpha-1} p(s) d s+c_{1}+c_{2} t, & t \in[0, \xi] ; \\
-\frac{1}{\Gamma(\alpha)} \int_{0}^{t}(t-s)^{\alpha-1} p(s) d s+c_{3}+c_{4} t, & t \in(\xi, 1] .\end{cases}
\end{aligned}
$$

Since $u^{\prime}(0)=0, \Delta u(\xi)=\int_{0}^{\xi} g(t) u(t) d t, \Delta u^{\prime}(\xi)=$ $\int_{0}^{\xi} h(t) u(t) d t$, and $u(1)=\int_{0}^{1} k(t) u(t) d t$, we know that

$$
\begin{aligned}
& c_{2}=0 ; \\
& c_{1}+\int_{0}^{\xi} g(t) u(t) d t=c_{3}+c_{4} \xi ; \\
& c_{2}+\int_{0}^{\xi} h(t) u(t) d t=c_{4} ; \\
& -\frac{1}{\Gamma(\alpha)} \int_{0}^{1}(1-s)^{\alpha-1} p(s) d s+c_{3}+c_{4} \\
& =\int_{0}^{1} k(t) u(t) d t .
\end{aligned}
$$


Then

$$
\begin{aligned}
c_{1}= & \frac{1}{\Gamma(\alpha)} \int_{0}^{1}(1-s)^{\alpha-1} p(s) d s-\int_{0}^{\xi} g(t) u(t) d t \\
& -(1-\xi) \int_{0}^{\xi} h(t) u(t) d t+\int_{0}^{1} k(t) u(t) d t \\
c_{2}= & 0 ; \\
c_{3}= & \frac{1}{\Gamma(\alpha)} \int_{0}^{1}(1-s)^{\alpha-1} p(s) d s-\int_{0}^{\xi} h(t) u(t) d t \\
& +\int_{0}^{1} k(t) u(t) d t ; \\
c_{4}= & \int_{0}^{\xi} h(t) u(t) d t
\end{aligned}
$$

that is, $u$ satisfies (25a) and (25b).

If (25a) and (25b) hold, by Lemma 6 we know that

$$
{ }^{c} D_{0}^{\alpha} u(t)={ }^{c} D_{0}^{\alpha} I_{0}^{\alpha} p(t) .
$$

Since $p(t) \in P C[0,1]$, we know that $I_{0}^{\alpha} p \in C[0,1]$ are continuous on $[0,1]$ as well as $I_{0}^{\alpha} p(0)=0$ and $\left[I_{0}^{\alpha} p\right]^{\prime}(0)=0$, so (29) turns to be

$$
{ }^{c} D_{0}^{\alpha} u(t)=-{ }^{c} D_{0}^{\alpha} I_{0}^{\alpha} p(t)=-{ }^{r l} D_{0}^{\alpha} I_{0}^{\alpha} p(t)=-p(t) .
$$

All the other conditions of (24) can be verified by direct computations and we omit it here.

Thirdly, we introduce some knowledge of order cone.

Definition 8. Let $X$ be a real Banach space and let $K$ be a subset of $X$. Then $K$ is called an order cone if

(i) $K$ is closed, nonempty, and $K \neq \theta$;

(ii) $a, b \in \mathbb{R}, a, b \geq 0, x, y \in K \Rightarrow a x+b y \in K$;

(iii) $x \in K$ and $-x \in K \Rightarrow x=\theta$.

On this basis, $u \in K$ is denoted by $u \geq \theta$, while $u>\theta$ means that $u \in K$ and $u \neq \theta$. Moreover, $K$ is called to be solid if $\operatorname{int}(K) \neq \phi$; that is, $K$ has interior points. $u \gg \theta$ means that $u$ is an interior point of $K$.

In this paper, we set

$$
X=\left\{u: u \in P C^{1}[0,1], \lim _{t \rightarrow 0_{+}} \frac{u^{\prime}(t)}{t^{\alpha-1}} \text { exist }\right\} .
$$

Then $X$ is a Banach space endowed with the norm

$$
\|u\|=\sup _{t \in[0,1]}|u(t)|+\sup _{t \in(0,1]}\left|\frac{u^{\prime}(t)}{t^{\alpha-1}}\right| .
$$

We set the cone

$$
K:=\left\{u \in X: u(t) \geq 0, u^{\prime}(t) \leq 0 \text { for } t \in[0,1]\right\} .
$$

Clearly $K$ is a solid cone in $X$. By the way, for any $u>\theta$, it is obvious that $u(0)>0$ and $u^{\prime}(0)=0$, which will be useful lately in this paper.

Fourthly, we introduce two lemmas which are very important in this paper.

Lemma 9 (see [17, Corollary 15.12]). We set

$$
\begin{aligned}
S_{+} & =\{(\mu, u) \in \mathbb{R} \times X:(\mu, u) \text { is a solution of } u \\
& =\mu(L u+N u) \text { with } \mu>0, u>\theta\} .
\end{aligned}
$$

If $\left(H_{1}\right)$ and $\left(H_{2}\right)$ are satisfied, then $\left(r(L)^{-1}, \theta\right)$ is a bifurcation point of $u=\mu(L u+N u)$ and $\overline{S_{+}}$contains an unbounded solution component $C_{+}\left(r(L)^{-1}\right)$ which passes through $\left(r(L)^{-1}, \theta\right)$.

If additionally $\left(\mathrm{H}_{3}\right)$ is satisfied, then $(\mu, u) \in C_{+}\left(r(L)^{-1}\right)$ and $\mu \neq r(L)^{-1}$ always imply that $\mu>0$ and $u>\theta$.

The conditions that Lemma 9 needs are stated as below.

$\left(H_{1}\right)$ The operators $L, N: X \rightarrow X$ are compact on the real Banach space $X . L+N$ is positive: that is, $(L+N) u \geq \theta$ when $u \geq \theta$. $L$ is linear and $\|N u\| /\|u\| \rightarrow 0$ as $\|u\| \rightarrow$ 0 . Moreover, $X$ has an order cone $K$ with $X=K-K$.

$\left(H_{2}\right)$ The spectral radius $r(L)$ of $L$ is positive.

$\left(H_{3}\right) L$ is strongly positive: that is, $L u \gg \theta$ for $u>\theta$.

Lemma 10 (see [18, Theorem 19.3]). Let $X$ be a Banach space and let $K \subset X$ be a solid cone. $L: X \rightarrow X$ is linear, compact, and strongly positive. Then we have the following:

(a) $r(L)>0, r(L)$ is a simple eigenvalue with an eigenvector $v \gg \theta$ and there is no other eigenvalue with positive eigenvector.

(b) For $y>\theta, \lambda \leq r(L)$, the equation $\lambda u-L u=y$ has no solution in $K$.

(c) Let $S: X \rightarrow X$ be a linear operator. If $S x-L x \geq \theta$ on $K$, then $r(s) \geq r(L)$, while $r(s)>r(L)$ if $S x-L x \gg \theta$ for $x>\theta$.

At last, we present here some conditions that we need in this paper.

$\left(C_{1}\right)$ At least one of the following two conditions is satisfied:

(1) $k(s)$ does not identically vanish on any subinterval of $[0,1]$

(2) $k(0)>0$.

$\left(C_{2}\right)$ There exist $a_{0}, a_{\infty} \in C([0,1],[0,+\infty))$ and $b_{0}, b_{\infty} \in$ $C([0,1],(-\infty, 0])$ such that

$$
\begin{array}{r}
\lim _{x, y \rightarrow 0} \frac{f(s, x, y)-a_{0}(s) x-b_{0}(s) y}{|x|+|y|}=0, \\
\lim _{x \geq 0, y \leq 0 ;|x|+|y| \rightarrow \infty} \frac{f(s, x, y)-a_{\infty}(s) x-b_{\infty}(s) y}{|x|+|y|}=0
\end{array}
$$

for all $s \in[0,1]$ uniformly. What is more, $a_{i}(0)>0$, $i=0, \infty$. 
$\left(C_{3}\right)$ There exists a function $\varphi \in C([0,1],[0,+\infty))$ such that

$$
f(s, x, y) \geq \varphi(s) x
$$

for all $(s, x, y) \in[0,1] \times[0,+\infty) \times(-\infty, 0]$. In addition, $\varphi(0)>0$.

\section{Property of $H, L_{0}, L_{\infty},(I-G)^{-1} L_{0}$ and $(I-G)^{-1} L_{\infty}$}

In this paper, we set

$$
\begin{aligned}
& (H u)(t)=-\frac{1}{\Gamma(\alpha)} \int_{0}^{t}(t-s)^{\alpha-1} f\left(s, u(s), u^{\prime}(s)\right) d s+\frac{1}{\Gamma(\alpha)} \int_{0}^{1}(1-s)^{\alpha-1} f\left(s, u(s), u^{\prime}(s)\right) d s, \quad t \in[0,1] ; \\
& (G u)(t)= \begin{cases}-\int_{0}^{\xi} g(s) u(s) d s-(1-\xi) \int_{0}^{\xi} h(s) u(s) d s+\int_{0}^{1} k(s) u(s) d s, \quad t \in[0, \xi] ; \\
-(1-t) \int_{0}^{\xi} h(s) u(s) d s+\int_{0}^{1} k(s) u(s) d s, & t \in(\xi, 1] ;\end{cases} \\
& \left(L_{0} u\right)(t)=-\frac{1}{\Gamma(\alpha)} \int_{0}^{t}(t-s)^{\alpha-1}\left[a_{0}(s) u(s)+b_{0}(s) u^{\prime}(s)\right] d s+\frac{1}{\Gamma(\alpha)} \int_{0}^{1}(1-s)^{\alpha-1}\left[a_{0}(s) u(s)+b_{0}(s) u^{\prime}(s)\right] d s, \\
& \left(L_{\infty} u\right)(t)=-\frac{1}{\Gamma(\alpha)} \int_{0}^{t}(t-s)^{\alpha-1}\left[a_{1}(s) u(s)+b_{\infty}(s) u^{\prime}(s)\right] d s+\frac{1}{\Gamma(\alpha)} \int_{0}^{1}(1-s)^{\alpha-1}\left[a_{\infty}(s) u(s)+b_{\infty}(s) u^{\prime}(s)\right] d s,
\end{aligned}
$$

Lemma 11. $H: X \rightarrow X$ is positive and compact.

Proof. Firstly, we will prove that $H u \in X$ for all $u \in X$. With all the other properties of $X$ easily verified, we only show here that $(H u)^{\prime}(t) / t^{\alpha-1}$ converges, while $t \rightarrow 0_{+}$.

We know that

$$
\begin{aligned}
& (H u)^{\prime}(t) \\
& \quad=-\frac{1}{\Gamma(\alpha-1)} \int_{0}^{t}(t-s)^{\alpha-2} f\left(s, u(s), u^{\prime}(s)\right) d s
\end{aligned}
$$

So,

$$
\begin{aligned}
& \lim _{t \rightarrow 0_{+}} \frac{(H u)^{\prime}(t)}{t^{\alpha-1}} \\
& =-\frac{1}{\Gamma(\alpha-1)} \lim _{t \rightarrow 0_{+}} \frac{\int_{0}^{t}(t-s)^{\alpha-2} f\left(s, u(s), u^{\prime}(s)\right) d s}{t^{\alpha-1}} \\
& =-\lim _{t \rightarrow 0_{+}} \frac{f\left(\tau, u(\tau), u^{\prime}(\tau)\right)}{\Gamma(\alpha-1)} \frac{\int_{0}^{t}(t-s)^{\alpha-2} d s}{t^{\alpha-1}}, \tau \in[0, t] \\
& =-\lim _{t \rightarrow 0_{+}} \frac{f\left(\tau, u(\tau), u^{\prime}(\tau)\right)}{\Gamma(\alpha)}, \quad \tau \in[0, t] \\
& =-\frac{f(0, u(0), 0)}{\Gamma(\alpha)} .
\end{aligned}
$$

Secondly, we show that $H$ is positive.
In fact, for $u \geq \theta$, observing the expression of $H u$, it is easy to see that $(H u)(t) \geq 0$ and $(H u)^{\prime}(t) \leq 0$ for $t \in[0,1]$.

Thirdly, we will prove that $H$ is compact. To this end, we only need to show that $\{(H u)(t): u \in D\}$ and $\left\{(H u)^{\prime}(t) / t^{\alpha-1}\right.$ : $u \in D\}$ are uniformly bounded and equicontinuous on $(0,1]$ for any bounded subset of $X$ named $D$. We only prove the later one since the proof of another one is easier.

Since $D$ is bounded, we can choose a constant number $M>0$ such that $\|u\| \leq M$ for all $u \in D$, which implies that $|u(t)| \leq M$ and $\left|u^{\prime}(t) / t^{\alpha-1}\right| \leq M$ and hence $\left|u^{\prime}(t)\right| \leq M$. Then there exists a constant number $\bar{M}$ such that $f\left(s, u(s), u^{\prime}(s)\right) \leq$ $\bar{M}$ for all $s \in[0,1], u \in D$. So we have

$$
\begin{aligned}
& \left|\frac{(H u)^{\prime}(t)}{t^{\alpha-1}}\right| \\
& =\frac{1}{\Gamma(\alpha-1)}\left|\int_{0}^{t} \frac{(t-s)^{\alpha-2} f\left(s, u(s), u^{\prime}(s)\right)}{t^{\alpha-1}} d s\right| \\
& \quad \leq \frac{\bar{M}}{\Gamma(\alpha-1)} \int_{0}^{t} \frac{(t-s)^{\alpha-2}}{t^{\alpha-1}} d s=\frac{\bar{M}}{\Gamma(\alpha)},
\end{aligned}
$$

which means that $\left\{(H u)^{\prime}(t) / t^{\alpha-1}: u \in D\right\}$ is uniformly bounded on $(0,1]$.

Next, we will prove that $\left\{(H u)^{\prime}(t) / t^{\alpha-1}: u \in D\right\}$ is equicontinuous on $(0,1]$. We know that

$$
\begin{aligned}
& \left|\frac{(H u)^{\prime}\left(t_{1}\right)}{t_{1}^{\alpha-1}}-\frac{(H u)^{\prime}\left(t_{2}\right)}{t_{1}^{\alpha-2}}\right| \\
& =\frac{1}{\Gamma(\alpha-1)} \mid \frac{\int_{0}^{t_{1}}\left(t_{1}-s\right)^{\alpha-2} f\left(s, u(s), u^{\prime}(s)\right) d s}{t_{1}^{\alpha-1}}
\end{aligned}
$$




$$
\begin{aligned}
& -\frac{\int_{0}^{t_{2}}\left(t_{2}-s\right)^{\alpha-2} f\left(s, u(s), u^{\prime}(s)\right) d s}{t_{2}^{\alpha-1}} \mid \\
& =\frac{1}{\Gamma(\alpha-1)} \mid \int_{0}^{1}(1-r)^{\alpha-2} \\
& \cdot f\left(t_{1} r, u\left(t_{1} r\right), u^{\prime}\left(t_{1} r\right)\right) d r-\int_{0}^{1}(1-r)^{\alpha-2} \\
& \cdot f\left(t_{2} r, u\left(t_{2} r\right), u^{\prime}\left(t_{2} r\right)\right) d r \mid \leq \frac{1}{\Gamma(\alpha-1)} \int_{0}^{1}(1 \\
& -r)^{\alpha-2} \mid f\left(t_{1} r, u\left(t_{1} r\right), u^{\prime}\left(t_{1} r\right)\right) \\
& -f\left(t_{2} r, u\left(t_{2} r\right), u^{\prime}\left(t_{2} r\right)\right) \mid d r ; \\
& \left|t_{1} r-t_{2} r\right| \leq\left|t_{1}-t_{2}\right| ; \\
& \left|u\left(t_{1} r\right)-u\left(t_{2} r\right)\right|=u^{\prime}\left(t_{*} r\right)\left|t_{1}-t_{2}\right| r \leq M\left|t_{1}-t_{2}\right|, \\
& \left|u^{\prime}\left(t_{1} r\right)-u^{\prime}\left(t_{2} r\right)\right| \leq\left|\frac{u^{\prime}\left(t_{1} r\right)}{\left(t_{1} r\right)^{\alpha-1} \mid}\right|\left(t_{1} r\right)^{\alpha-1}+\left|\frac{u^{\prime}\left(t_{2} r\right)}{\left(t_{2} r\right)^{\alpha-1}}\right| \\
& \cdot\left(t_{2} r\right)^{\alpha-1} \leq M\left(t_{1}^{\alpha-1}+t_{2}^{\alpha-1}\right) .
\end{aligned}
$$

By the uniform continuity of $f$ on $[0,1] \times[-M, M] \times$ $[-M, M]$ and (41), we know that, for any $\varepsilon>0$, there exists $\delta_{1}>0$ such that $t_{1}, t_{2} \in\left(0, \delta_{1}\right]$ implies

$$
\left|\frac{(H u)^{\prime}\left(t_{1}\right)}{t_{1}^{\alpha-1}}-\frac{(H u)^{\prime}\left(t_{2}\right)}{t_{1}^{\alpha-2}}\right| \leq \varepsilon .
$$

Moreover, when $\delta_{1} / 2 \leq t_{1}<t_{2} \leq 1$, we have that

$$
\begin{aligned}
& \left|\frac{(H u)^{\prime}\left(t_{2}\right)}{t_{2}^{\alpha-1}}-\frac{(H u)^{\prime}\left(t_{1}\right)}{t_{1}^{\alpha-1}}\right| \\
& =\frac{1}{\Gamma(\alpha-1)} \mid \frac{\int_{0}^{t_{2}}\left(t_{2}-s\right)^{\alpha-2} f\left(s, u(s), u^{\prime}(s)\right) d s}{t_{2}^{\alpha-1}} \\
& \quad-\frac{\int_{0}^{t_{1}}\left(t_{1}-s\right)^{\alpha-2} f\left(s, u(s), u^{\prime}(s)\right) d s}{t_{1}^{\alpha-1}} \mid \\
& =\frac{1}{\Gamma(\alpha-1)} \mid \frac{\int_{t_{1}}^{t_{2}}\left(t_{2}-s\right)^{\alpha-2} f\left(s, u(s), u^{\prime}(s)\right) d s}{t_{2}^{\alpha-1}} \\
& +\int_{0}^{t_{1}}\left[\frac{\left(t_{2}-s\right)^{\alpha-2}}{t_{2}^{\alpha-1}}-\frac{\left(t_{1}-s\right)^{\alpha-2}}{t_{1}^{\alpha-1}}\right] \\
& \quad \cdot f\left(s, u(s), u^{\prime}(s)\right) d s \mid
\end{aligned}
$$

$$
\begin{aligned}
& \leq \frac{\bar{M}}{\Gamma(\alpha-1)}\left[\frac{\int_{t_{1}}^{t_{2}}\left(t_{2}-s\right)^{\alpha-2} d s}{t_{2}^{\alpha-1}}\right. \\
& \left.+\int_{0}^{t_{1}}\left(\frac{\left(t_{1}-s\right)^{\alpha-2}}{t_{1}^{\alpha-1}}-\frac{\left(t_{2}-s\right)^{\alpha-2}}{t_{2}^{\alpha-1}}\right) d s\right] \\
& =\frac{2 \bar{M}\left(t_{2}-t_{1}\right)^{\alpha-1}}{\Gamma(\alpha) t_{2}^{\alpha-1}} \leq \frac{2^{\alpha} \bar{M}}{\Gamma(\alpha) \delta_{1}^{\alpha-1}}\left(t_{2}-t_{1}\right)^{\alpha-1} .
\end{aligned}
$$

So

$$
\begin{aligned}
& \left|t_{2}-t_{1}\right|<\left[\frac{\Gamma(\alpha) \delta_{1}^{\alpha-1} \varepsilon}{2^{\alpha} \bar{M}}\right]^{1 /(\alpha-1)} \\
& \text { will lead to }\left|\frac{(H u)^{\prime}\left(t_{2}\right)}{t_{2}^{\alpha-1}}-\frac{(H u)^{\prime}\left(t_{1}\right)}{t_{1}^{\alpha-1}}\right| \leq \varepsilon, \\
& \qquad t_{1}, t_{2} \in\left[\frac{\delta_{1}}{2}, 1\right] .
\end{aligned}
$$

We set $\delta=\min \left\{\delta_{1} / 2,\left[\Gamma(\alpha) \delta_{1}^{\alpha-1} \varepsilon / 2^{\alpha} \bar{M}\right]^{1 /(\alpha-1)}\right\}$; by (42) and (44), we know that $\left|(H u)^{\prime}\left(t_{2}\right) / t_{2}^{\alpha-1}-(H u)^{\prime}\left(t_{1}\right) / t_{1}^{\alpha-1}\right| \leq \varepsilon$ for all $u \in D$ and $t_{1}, t_{2} \in(0,1]$ satisfy $\left|t_{2}-t_{1}\right|<\delta$.

Lemma 12. $L_{0}, L_{\infty}: X \rightarrow X$ are linear, compact, and positive.

Proof. Similar to Lemma 11, we can verify that $L_{0}, L_{\infty}: X \rightarrow$ $X$ are compact and positive. Moreover, they are obviously linear operators.

Lemma 13. If $\left(C_{1}\right)$ holds and $\|G\|<1$, then $(I-G)^{-1} L_{0}$ and $(I-G)^{-1} L_{\infty}$ are linear, compact, and strongly positive.

Proof. It is obvious that $G: X \rightarrow X$ is a positive linear operator. So if $\|G\|<1, I-G$ will have a positive linear bounded inverse operator:

$$
(I-G)^{-1}=I+G+G^{2}+\cdots .
$$

Since $L_{0}$ and $L_{\infty}$ are compact, we know that $(I-G)^{-1} L_{0}$ and $(I-G)^{-1} L_{\infty}$ are compact. In the next, we will only prove that $(I-G)^{-1} L_{0}$ is strongly positive since the proof for another one is similar.

For any given $x \in X, x>\theta$, if we set

$$
y=(I-G)^{-1} L_{0} x,
$$

we will know that $y \geq L_{0} x>\theta$ (noticing the expression of $L_{0}$, we can see that $L_{0}$ will turn any positive element of $X$ to be a positive element of $X$ ). By (46), we know that

$$
(I-G) y=L_{0} x
$$

and hence

$$
y=G y+L_{0} x .
$$


Since $y>\theta$, we know that $y^{\prime}(t) \leq 0$ for $0 \in[0,1]$, so by $\left(C_{1}\right)$ and (48), we will know that

$$
\begin{aligned}
y(t) & \geq y(1)=(G y)(1)+\left(L_{0} x\right)(1) \\
& =\int_{0}^{1} k(s) y(s) d s>0 .
\end{aligned}
$$

On the other hand, for $t \in(0,1]$, by $\left(C_{2}\right)$ and $u(0)>0$, we will know that

$$
\begin{aligned}
& y^{\prime}(t)=(G y)^{\prime}(t)+\left(L_{0} x\right)^{\prime}(t) \leq\left(L_{0} x\right)^{\prime}(t) \\
& \quad=-\frac{1}{\Gamma(\alpha-1)} \\
& \cdot \int_{0}^{t}(t-s)^{\alpha-2}\left[a_{0}(s) u(s)+b_{0}(s) u^{\prime}(s)\right] d s \\
& \leq-\frac{1}{\Gamma(\alpha-1)} \int_{0}^{t}(t-s)^{\alpha-2} a_{0}(s) u(s) d s<0 .
\end{aligned}
$$

What is more, similar to (39), we have

$$
\begin{aligned}
& \lim _{t \rightarrow 0_{+}} \frac{y^{\prime}(t)}{t^{\alpha-1}}=-\frac{1}{\Gamma(\alpha-1)} \lim _{t \rightarrow 0_{+}} \frac{1}{t^{\alpha-1}} \\
& \quad \cdot \int_{0}^{t}(t-s)^{\alpha-2}\left[a_{0}(s) u(s)+b_{0}(s) u^{\prime}(s)\right] d s \\
& \quad=-\frac{a_{0}(0) u(0)}{\Gamma(\alpha)}<0 .
\end{aligned}
$$

By (50) and (51) and the piecewise continuity of $y^{\prime}(t)$ on $[0,1]$, we know that there must exist a positive number $\tau$ such that

$$
\sup _{t \in(0,1]} \frac{y^{\prime}(t)}{t^{\alpha-1}}<-\tau
$$

Equations (49) and (52) exactly mean that $y \gg \theta$.

\section{Main Results and the Proof}

Theorem 14. Suppose that $\left(C_{1}\right)-\left(C_{3}\right)$ hold and $\|G\|<1$; then there must exist at least one positive solution of (2) if $1 / r((I-$ $\left.G)^{-1} L_{\infty}\right)<a<1 / r\left((I-G)^{-1} L_{0}\right)$ or $1 / r\left((I-G)^{-1} L_{\infty}\right)>a>$ $1 / r\left((I-G)^{-1} L_{0}\right)$.

Proof. The proof is divided into three parts.

In Part 1, we consider the auxiliary equation (see (53)) whose solutions of the kind $\mu=1$ will be the solutions of (2) and we transform it into a functional operator equation (see (57)).

In Part 2, we will verify that $(I-G)^{-1} L_{0},(I-G)^{-1} N_{0}$ satisfy all the conditions required to apply Lemmas 9 and 10, where $N_{0}$ will be defined in Part 1 .
In Part 3, we apply Lemmas 9 and 10 to get the existence of at least one positive solution of (2).

Part 1. We consider the following problem.

$$
\begin{aligned}
{ }^{c} D_{0_{+}}^{\alpha} u(t)+\mu a f\left(t, u(t), u^{\prime}(t)\right) & =0, \quad t \in(0,1), \\
u^{\prime}(0) & =0, \\
u(1) & =\int_{0}^{1} k(t) u(t) d t, \\
\Delta u(\xi) & =\int_{0}^{\xi} g(t) u(t) d t, \\
\Delta u^{\prime}(\xi) & =\int_{0}^{\xi} h(t) u(t) d t .
\end{aligned}
$$

We call $(\mu, u) \in \mathbb{R} \times X$ to be a solution of (53) if it satisfies (53). It is clear that any solution of $(53)$ of the form $(1, u)$ yields a solution $u$ of (2).

Due to Lemma $7,(\mu, u) \in \mathbb{R} \times X$ is a solution of (53) if and only if

$$
u=\mu a H u+G u
$$

that is,

$$
u=\mu a(I-G)^{-1} H u
$$

If we set

$$
N_{0} u=H u-L_{0} u,
$$

then $u$ is a solution of (53) if and only if

$$
u=\mu a\left[(I-G)^{-1} L_{0} u+(I-G)^{-1} N_{0} u\right] .
$$

Part 2. By Lemmas $11-13$, we have confirmed that (I$G)^{-1} H,(I-G)^{-1} L_{0},(I-G)^{-1} N_{0}$ are compact, $(I-G)^{-1} H$ is positive, and $(I-G)^{-1} L_{0}$ is strongly positive. Now we only need to verify that

$$
\lim _{\|u\| \rightarrow 0} \frac{\left\|(I-G)^{-1} N_{0} u\right\|}{\|u\|}=0 .
$$

$\forall \varepsilon>0$, by $\left(C_{2}\right)$, there must exist a number $\delta>0$ such that $|x|<\delta$ and $|y|<\delta$ imply

$$
\left|\frac{f(s, x, y)-a_{0}(s) x-b_{0}(s) y}{|x|+|y|}\right|<\frac{\Gamma(\alpha+1)}{2} \varepsilon \text {. }
$$

Then, if $\|u\|<\delta$, there will be

$$
\frac{\left|\left(N_{0} u\right)(t)\right|}{\|u\|}=\mid-\frac{1}{\Gamma(\alpha)} \int_{0}^{t} \frac{(t-s)^{\alpha-1}\left[f\left(s, u(s), u^{\prime}(s)\right)-a_{0}(s) u(s)-b_{0}(s) u^{\prime}(s)\right]}{\|u\|} d s
$$




$$
\begin{aligned}
& +\frac{1}{\Gamma(\alpha)} \int_{0}^{1} \frac{(1-s)^{\alpha-1}\left[f\left(s, u(s), u^{\prime}(s)\right)-a_{0}(s) u(s)-b_{0}(s) u^{\prime}(s)\right]}{\|u\|} d s \mid \leq \frac{1}{\Gamma(\alpha)} \\
& \cdot \int_{0}^{t}(t-s)^{\alpha-1} \frac{\left|f\left(s, u(s), u^{\prime}(s)\right)-a_{0}(s) u(s)-b_{0}(s) u^{\prime}(s)\right|}{\|u\|} d s+\frac{1}{\Gamma(\alpha)} \\
& \cdot \int_{0}^{1}(1-s)^{\alpha-1} \frac{\left|f\left(s, u(s), u^{\prime}(s)\right)-a_{0}(s) u(s)-b_{0}(s) u^{\prime}(s)\right|}{\|u\|} d s \leq \frac{1}{\Gamma(\alpha)} \\
& \cdot \int_{0}^{t}(t-s)^{\alpha-1} \frac{\left|f\left(s, u(s), u^{\prime}(s)\right)-a_{0}(s) u(s)-b_{0}(s) u^{\prime}(s)\right|}{|u(s)|+\left|u^{\prime}(s)\right|} d s+\frac{1}{\Gamma(\alpha)} \\
& \cdot \int_{0}^{1}(1-s)^{\alpha-1} \frac{\left|f\left(s, u(s), u^{\prime}(s)\right)-a_{0}(s) u(s)-b_{0}(s) u^{\prime}(s)\right|}{|u(s)|+\left|u^{\prime}(s)\right|} d s \leq \frac{\alpha \varepsilon}{2} \int_{0}^{t}(t-s)^{\alpha-1} d s+\frac{\alpha \varepsilon}{2} \int_{0}^{1}(1-s)^{\alpha-1} d s=\frac{\varepsilon t^{\alpha}}{2} \\
& +\frac{\varepsilon}{2} \leq \varepsilon .
\end{aligned}
$$

Moreover, we know that

$$
\begin{aligned}
\left|\frac{\left(N_{0} u\right)^{\prime}(t)}{t^{\alpha-1}\|u\|}\right| & =\frac{1}{\Gamma(\alpha-1)}\left|\int_{0}^{t} \frac{(t-s)^{\alpha-2}\left[f\left(s, u(s), u^{\prime}(s)\right)-a_{0}(s) u(s)-b_{0}(s) u^{\prime}(s)\right]}{t^{\alpha-1}\|u\|} d s\right| \\
& \leq \frac{1}{\Gamma(\alpha-1)} \int_{0}^{t} \frac{(t-s)^{\alpha-2}\left|f\left(s, u(s), u^{\prime}(s)\right)-a_{0}(s) u(s)-b_{0}(s) u^{\prime}(s)\right|}{t^{\alpha-1}\|u\|} d s \\
& \leq \frac{1}{\Gamma(\alpha-1)} \int_{0}^{t} \frac{(t-s)^{\alpha-2}}{t^{\alpha-1}} \frac{\left|f\left(s, u(s), u^{\prime}(s)\right)-a_{0}(s) u(s)-b_{0}(s) u^{\prime}(s)\right|}{|u(s)|+\left|u^{\prime}(s)\right|} d s \\
& \leq \frac{1}{\Gamma(\alpha-1)} \frac{\Gamma(\alpha+1)}{2} \varepsilon \int_{0}^{t} \frac{(t-s)^{\alpha-2}}{t^{\alpha-1}} d s=\frac{1}{\Gamma(\alpha-1)} \frac{\Gamma(\alpha+1)}{2} \varepsilon \frac{1}{\alpha-1}=\frac{\alpha \varepsilon}{2} \leq \varepsilon .
\end{aligned}
$$

By (60) and (61), we will know that

$$
\lim _{\|u\| \rightarrow 0} \frac{\left\|N_{0} u\right\|}{\|u\|}=0 .
$$

Equation (62) together with the boundedness of $(I-G)^{-1}$ will lead to

$$
\lim _{\|u\| \rightarrow 0} \frac{\left\|(I-G)^{-1} N_{0} u\right\|}{\|u\|} \longrightarrow 0
$$

Part 3. Applying Lemmas 9 and 10, we can draw a conclusion as below.

For (57), from $\left(1 / \operatorname{ar}\left((I-G)^{-1} L_{0}\right), \theta\right)$ there emanates an unbounded continua of positive solutions $C_{+} \subset \bar{D}_{+}$, where

$$
\begin{aligned}
D_{+} & =\left\{(\mu, u) \in \mathbb{R} \times X: u=\mu a(I-G)^{-1} H u \text { with } \mu\right. \\
& >0, u>\theta\} .
\end{aligned}
$$

Furthermore, $(\mu, u) \in C_{+}$and $\mu \neq 1 / \operatorname{ar}\left((I-G)^{-1} L_{0}\right)$ always imply that $u>\theta$.

To verify the existence of at least one positive solution of (2), we only need to show that $C_{+}$crosses the hyperplane $\{1\} \times$ $X$ in $\mathbb{R} \times X$. To this end, it will be enough to show that $C_{+}$joins $\left(1 / \operatorname{ar}\left((I-G)^{-1} L_{0}\right), \theta\right)$ to $\left(1 / \operatorname{ar}\left((I-G)^{-1} L_{\infty}\right),+\infty\right)$.

Suppose that $\left(\mu_{n}, u_{n}\right) \in C_{+}$satisfy $\mu_{n}+\left\|u_{n}\right\| \rightarrow \infty$. To begin with, we will show that $\left\{\mu_{n}\right\}$ is bounded. Defining $L_{\varphi}$ as

$$
\begin{aligned}
\left(L_{\varphi} u\right)(t)= & -\frac{1}{\Gamma(\alpha)} \int_{0}^{t}(t-s)^{\alpha-1} \varphi(s) u(s) d s \\
& +\frac{1}{\Gamma(\alpha)} \int_{0}^{1}(1-s)^{\alpha-1} \varphi(s) u(s) d s, \\
& t \in[0,1],
\end{aligned}
$$

similar to Lemma 13 , we can verify that $(I-G)^{-1} L_{\varphi}$ : $K \rightarrow K$ is linear, compact, and strongly positive. Then, 
due to Lemma 10(b), we see that there is a contradiction for sufficiently large $n$ in the following inequality:

$$
u_{n}=\mu_{n} a(I-G)^{-1} H u_{n} \geq \mu_{n} a(I-G)^{-1} L_{\varphi} u_{n} .
$$

Then, $\left\{\mu_{n}\right\}$ is bounded and hence $\lim _{n \rightarrow \infty}\left\|u_{n}\right\| \rightarrow \infty$.

We choose a subsequence of $\left\{\mu_{n}\right\}$ converging to $\mu_{*}$. Without loss of generality, we relabel the subsequence to be $\left\{\mu_{n}\right\}$ just for convenience.

Now we set

$$
N_{\infty} u=H u-L_{\infty} u
$$

then

$$
\begin{aligned}
v_{n} & =\frac{u_{n}}{\left\|u_{n}\right\|} \\
& =\frac{\mu_{n} a(I-G)^{-1} L_{\infty} u_{n}}{\left\|u_{n}\right\|}+\frac{\mu_{n} a(I-G)^{-1} N_{\infty} u_{n}}{\left\|u_{n}\right\|} \\
& =\mu_{n} a(I-G)^{-1} L_{\infty} v_{n}+\mu_{n} a \frac{(I-G)^{-1} N_{\infty} u_{n}}{\left\|u_{n}\right\|} .
\end{aligned}
$$

Since $(I-G)^{-1} L_{\infty}$ is compact, we can find a subsequence of $\left\{(I-G)^{-1} L_{\infty} v_{n}\right\}$ named $\left\{(I-G)^{-1} L_{\infty} v_{n_{k}}\right\}$ converging to $v_{0} \in$ $X$. Moreover, we know that

$$
\lim _{u \in K,\|u\| \rightarrow \infty} \frac{(I-G)^{-1} N_{\infty} u}{\|u\|}=\theta
$$

(see the proof in the appendix),

so

$$
\lim _{k \rightarrow \infty} v_{n_{k}}=\mu_{*} a v_{0}:=v_{*} .
$$

It is obvious that $v_{*}>\theta$ and $\left\|v_{*}\right\|=1$. Let $k \rightarrow \infty$ in $v_{n_{k}}=$ $\mu_{n_{k}} a(I-G)^{-1} L_{\infty} v_{n_{k}}+\mu_{n_{k}} a\left((I-G)^{-1} N_{\infty} u_{n_{k}} /\left\|u_{n_{k}}\right\|\right)$; we know that

$$
v_{*}=\mu_{*} a(I-G)^{-1} L_{\infty} v_{*} .
$$

Since $(I-G)^{-1} L_{\infty}: X \rightarrow X$ is linear, compact, and strongly positive, by Lemma 10 and (71) we know that

$$
\mu_{*}=\frac{1}{\operatorname{ar}\left((I-G)^{-1} L_{\infty}\right)},
$$

which implies that $C_{+}$joins $\left(1 / \operatorname{ar}\left((I-G)^{-1} L_{0}\right), \theta\right)$ to $(1 / \operatorname{ar}((I-$ $\left.\left.G)^{-1} L_{\infty}\right),+\infty\right)$.

As a corollary of Theorem 14, we have the following conclusion.

Theorem 15. Suppose that $\left(C_{1}\right)-\left(C_{3}\right)$ hold. If

$$
\begin{aligned}
-\int_{0}^{\xi} g(s) d s-(1-\xi) \int_{0}^{\xi} h(s) d s & +\int_{0}^{1} k(s) d s<1, \\
& -\int_{0}^{\xi} h(s) d s<\xi^{\alpha-1},
\end{aligned}
$$

then there must exist at least one positive solution of (2) when $1 / r\left((I-G)^{-1} L_{\infty}\right)<a<1 / r\left((I-G)^{-1} L_{0}\right)$ or $1 / r((I-$ $\left.G)^{-1} L_{\infty}\right)>a>1 / r\left((I-G)^{-1} L_{0}\right)$.
Proof. With all the other conditions of Theorem 14 satisfied, we only need to verify that $\|G\|<1$. For any $u \in X$ and $t \in$ $[0, \xi]$, we have

$$
\begin{aligned}
& |(G u)(t)|=\mid-\int_{0}^{\xi} g(s) u(s) d s \\
& -(1-\xi) \int_{0}^{\xi} h(s) u(s) d s+\int_{0}^{1} k(s) u(s) d s \mid \\
& \leq-\int_{0}^{\xi} g(s)|u(s)| d s-(1-\xi) \int_{0}^{\xi} h(s)|u(s)| d s \\
& +\int_{0}^{1} k(s)|u(s)| d s \leq\left[-\int_{0}^{\xi} g(s) d s\right. \\
& \left.-(1-\xi) \int_{0}^{\xi} h(s) d s+\int_{0}^{1} k(s) d s\right]\|u\| .
\end{aligned}
$$

For any $u \in X$ and $t \in(\xi, 1]$, we have

$|(G u)(t)|$

$$
\begin{aligned}
& =\left|-(1-t) \int_{0}^{\xi} h(s) u(s) d s+\int_{0}^{1} k(s) u(s) d s\right| \\
& \leq-(1-\xi) \int_{0}^{\xi} h(s)|u(s)| d s+\int_{0}^{1} k(s)|u(s)| d s \\
& \leq\left[-(1-\xi) \int_{0}^{\xi} h(s) d s+\int_{0}^{1} k(s) d s\right]\|u\| .
\end{aligned}
$$

For any $u \in X$ and $t \in[0, \xi]$, we have

$$
(G u)^{\prime}(t)=0 .
$$

For any $u \in X$ and $t \in(\xi, 1]$, we have

$$
\begin{aligned}
\left|\frac{(G u)^{\prime}(t)}{t^{\alpha-1}}\right| & =\left|\frac{\int_{0}^{\xi} h(s) u(s) d s}{t^{\alpha-1}}\right| \leq-\frac{\int_{0}^{\xi} h(s)|u(s)| d s}{t^{\alpha-1}} \\
& <-\frac{\int_{0}^{\xi} h(s) d s}{\xi^{\alpha-1}}\|u\| .
\end{aligned}
$$

By (74)-(77), we know that $\|G\|<1$.

\section{Example}

Example 1. Consider the following problem.

$$
\begin{array}{r}
{ }^{c} D_{0_{+}}^{3 / 2} u(t)+a\left[2 u(t)+e^{u^{\prime}(t)} \sin u(t)-u^{\prime}(t)\right]=0, \\
t \in[0,1],
\end{array}
$$




$$
\begin{aligned}
& u^{\prime}(0)=0, \\
& u(1)=\int_{0}^{1}(1-t) u(t) d t \\
& \Delta u^{\prime}\left(\frac{1}{2}\right)=-\int_{0}^{1 / 2} t u(t) d t \\
& \Delta u\left(\frac{1}{2}\right)=-\int_{0}^{1 / 2} t^{2} u(t) d t \\
& \left(L_{0} u\right)(t)=-\frac{1}{\Gamma(3 / 2)} \int_{0}^{t}(t-s)^{\alpha-1}\left[3 u(s)-u^{\prime}(s)\right] d s+\frac{1}{\Gamma(3 / 2)} \int_{0}^{1}(1-s)^{\alpha-1}\left[3 u(s)-u^{\prime}(s)\right] d s ; \\
& \left(L_{\infty} u\right)(t)=-\frac{1}{\Gamma(3 / 2)} \int_{0}^{t}(t-s)^{\alpha-1}\left[2 u(s)-u^{\prime}(s)\right] d s+\frac{1}{\Gamma(3 / 2)} \int_{0}^{1}(1-s)^{\alpha-1}\left[2 u(s)-u^{\prime}(s)\right] d s ; \\
& (G u)(t)= \begin{cases}\int_{0}^{1 / 2} s^{2} u(s) d s+\frac{1}{2} \int_{0}^{1 / 2} s u(s) d s+\int_{0}^{1}(1-s) u(s) d s, & t \in\left[0, \frac{1}{2}\right] \\
(1-t) \int_{0}^{1 / 2} s u(s) d s+\int_{0}^{1}(1-s) u(s) d s, & t \in\left(\frac{1}{2}, 1\right]\end{cases}
\end{aligned}
$$

then there must be at least one positive solution of $\left(E_{1}\right)$ when $1 / r\left((I-G)^{-1} L_{\infty}\right)>a>1 / r(I-G)^{-1}\left(L_{0}\right)$. (We can verify that $r\left((I-G)^{-1} L_{0}\right) \geq r\left((I-G)^{-1} L_{\infty}\right)$ by Lemma 10.)

Proof. Let $\alpha=3 / 2, \xi=1 / 2, f(s, x, y)=2 x+e^{y} \sin x-y$, $g(s)=-s^{2}, h(s)=-s, k(s)=1-s$; then $\left(E_{1}\right)$ turns to be (2). Now we set $a_{0}(s) \equiv 3, b_{0}(s) \equiv-1, a_{\infty}(s) \equiv 2$, $b_{\infty}(s) \equiv-1, \varphi(s) \equiv 1$. We can verify that $\left(C_{1}\right)-\left(C_{3}\right)$ hold. Then by Theorem 15 we know that there must be at least one positive solution of $\left(E_{1}\right)$ when $1 / r\left((I-G)^{-1} L_{\infty}\right)>a>$ $1 / r(I-G)^{-1}\left(L_{0}\right)$.

\section{Appendix}

At the very first, we recall that

$$
\begin{gathered}
\left(N_{\infty} u\right)(t)=-\frac{1}{\Gamma(\alpha)} \int_{0}^{t}(t-s)^{\alpha-1}\left[f\left(s, u(s), u^{\prime}(s)\right)\right. \\
\left.-a_{\infty}(s) u(s)-b_{\infty}(s) u^{\prime}(s)\right] d s+\frac{1}{\Gamma(\alpha)}
\end{gathered}
$$

$$
\begin{aligned}
& \cdot \int_{0}^{1}(1-s)^{\alpha-1}\left[f\left(s, u(s), u^{\prime}(s)\right)-a_{\infty}(s) u(s)\right. \\
& \left.-b_{\infty}(s) u^{\prime}(s)\right] d s \\
& \left(N_{\infty} u\right)^{\prime}(t)=-\frac{1}{\Gamma(\alpha-1)} \int_{0}^{t}(t-s)^{\alpha-2} \\
& \cdot\left[f\left(s, u(s), u^{\prime}(s)\right)-a_{\infty}(s) u(s)\right. \\
& \left.-b_{\infty}(s) u^{\prime}(s)\right] d s .
\end{aligned}
$$

For any $\varepsilon>0$ and $u \in K$, since

$$
\lim _{x \geq 0, y \leq 0 ;|x|+|y| \rightarrow \infty} \frac{f(s, x, y)-a_{\infty}(s) x-b_{\infty}(s) y}{|x|+|y|}=0
$$

for all $s \in[0,1]$ uniformly, similar to (60)-(61) we can find sufficiently large number $M_{1}$ such that

$$
\begin{aligned}
& \frac{1}{\Gamma(\alpha)} \int_{I_{u} \cap[0, t]}(t-s)^{\alpha-1} \frac{\left|f\left(s, u(s), u^{\prime}(s)\right)-a_{\infty}(s) u(s)-b_{\infty}(s) u^{\prime}(s)\right|}{|u(s)|+\left|u^{\prime}(s)\right|} d s \\
& +\frac{1}{\Gamma(\alpha)} \int_{I_{u} \cap[0,1]}(1-s)^{\alpha-1} \frac{\left|f\left(s, u(s), u^{\prime}(s)\right)-a_{\infty}(s) u(s)-b_{\infty}(s) u^{\prime}(s)\right|}{|u(s)|+\left|u^{\prime}(s)\right|} d s \leq \frac{\varepsilon}{2}, \\
& \frac{1}{\Gamma(\alpha-1)} \int_{I_{u} \cap[0, t]} \frac{(t-s)^{\alpha-2}}{t^{\alpha-1}} \frac{\left|f\left(s, u(s), u^{\prime}(s)\right)-a_{\infty}(s) u(s)-b_{\infty}(s) u^{\prime}(s)\right|}{|u(s)|+\left|u^{\prime}(s)\right|} d s \leq \frac{\varepsilon}{2},
\end{aligned}
$$

where

$$
I_{u}=\left\{0 \leq s \leq 1:|u(s)|+\left|u^{\prime}(s)\right|>M_{1}\right\} .
$$

What is more, because $f(s, x, y)-a_{\infty}(s) x-b_{\infty}(s) y$ is bounded on $\left\{(s, x, y): s \in[0,1],|x|+|y| \leq M_{1}\right\}$, we can find sufficiently large $M_{2}$ such that 


$$
\begin{aligned}
& \frac{1}{\Gamma(\alpha)} \int_{J_{u} \cap[0, t]}(t-s)^{\alpha-1} \frac{\left|f\left(s, u(s), u^{\prime}(s)\right)-a_{\infty}(s) u(s)-b_{\infty}(s) u^{\prime}(s)\right|}{M_{2}} d s \\
& +\frac{1}{\Gamma(\alpha)} \int_{J_{u} \cap[0,1]}(1-s)^{\alpha-1} \frac{\left|f\left(s, u(s), u^{\prime}(s)\right)-a_{\infty}(s) u(s)-b_{\infty}(s) u^{\prime}(s)\right|}{M_{2}} d s \leq \frac{\varepsilon}{2}, \\
& \frac{1}{\Gamma(\alpha-1)} \int_{J_{u} \cap[0, t]} \frac{(t-s)^{\alpha-2}}{t^{\alpha-1}} \frac{\left|f\left(s, u(s), u^{\prime}(s)\right)-a_{\infty}(s) u(s)-b_{\infty}(s) u^{\prime}(s)\right|}{M_{2}} d s \leq \frac{\varepsilon}{2},
\end{aligned}
$$

where

$$
J_{u}=\left\{0 \leq s \leq 1:|u(s)|+\left|u^{\prime}(s)\right| \leq M_{1}\right\} .
$$

So, by (A.3) and (A.6), we know that, for $u \in K$ with $\|u\| \geq$ $M_{2}$, there must be

$$
\begin{aligned}
& \cdot \frac{\left|f\left(s, u(s), u^{\prime}(s)\right)-a_{\infty}(s) u(s)-b_{\infty}(s) u^{\prime}(s)\right|}{\|u\|} d s \\
& +\frac{1}{\Gamma(\alpha)} \int_{J_{u} \cap[0,1]}(1-s)^{\alpha-1}
\end{aligned}
$$

$$
\begin{aligned}
& \cdot \frac{\left|f\left(s, u(s), u^{\prime}(s)\right)-a_{\infty}(s) u(s)-b_{\infty}(s) u^{\prime}(s)\right|}{\|u\|} d s \\
& \leq \frac{1}{\Gamma(\alpha)} \int_{I_{u} \cap[0, t]}(t-s)^{\alpha-1}
\end{aligned}
$$$$
\frac{\left|\left[N_{\infty}(u)\right](t)\right|}{\|u\|} \leq \frac{1}{\Gamma(\alpha)} \int_{0}^{t}(t-s)^{\alpha-1}
$$$$
\frac{\left|f\left(s, u(s), u^{\prime}(s)\right)-a_{\infty}(s) u(s)-b_{\infty}(s) u^{\prime}(s)\right|}{\|u\|} d s
$$$$
\cdot \frac{\left|f\left(s, u(s), u^{\prime}(s)\right)-a_{\infty}(s) u(s)-b_{\infty}(s) u^{\prime}(s)\right|}{|u(s)|+\left|u^{\prime}(s)\right|} d s
$$$$
+\frac{1}{\Gamma(\alpha)} \int_{I_{u} \cap[0,1]}(1-s)^{\alpha-1}
$$$$
+\frac{1}{\Gamma(\alpha)} \int_{0}^{1}(1-s)^{\alpha-1}
$$$$
\cdot \frac{\left|f\left(s, u(s), u^{\prime}(s)\right)-a_{\infty}(s) u(s)-b_{\infty}(s) u^{\prime}(s)\right|}{|u(s)|+\left|u^{\prime}(s)\right|} d s
$$$$
\cdot \frac{\left|f\left(s, u(s), u^{\prime}(s)\right)-a_{\infty}(s) u(s)-b_{\infty}(s) u^{\prime}(s)\right|}{\|u\|} d s
$$$$
+\frac{1}{\Gamma(\alpha)} \int_{J_{u} \cap[0, t]}(t-s)^{\alpha-1}
$$$$
=\frac{1}{\Gamma(\alpha)} \int_{I_{u} \cap[0, t]}(t-s)^{\alpha-1}
$$$$
\text { . } \frac{\left|f\left(s, u(s), u^{\prime}(s)\right)-a_{\infty}(s) u(s)-b_{\infty}(s) u^{\prime}(s)\right|}{M_{2}} d s
$$$$
\frac{\left|f\left(s, u(s), u^{\prime}(s)\right)-a_{\infty}(s) u(s)-b_{\infty}(s) u^{\prime}(s)\right|}{\|u\|} d s
$$$$
+\frac{1}{\Gamma(\alpha)} \int_{J_{u} \cap[0,1]}(1-s)^{\alpha-1}
$$$$
+\frac{1}{\Gamma(\alpha)} \int_{I_{u} \cap[0,1]}(1-s)^{\alpha-1}
$$$$
\text { . } \frac{\left|f\left(s, u(s), u^{\prime}(s)\right)-a_{\infty}(s) u(s)-b_{\infty}(s) u^{\prime}(s)\right|}{M_{2}} d s
$$

$\cdot \frac{\left|f\left(s, u(s), u^{\prime}(s)\right)-a_{\infty}(s) u(s)-b_{\infty}(s) u^{\prime}(s)\right|}{\|u\|} d s$

$\leq \varepsilon$.

$$
+\frac{1}{\Gamma(\alpha)} \int_{J_{u} \cap[0, t]}(t-s)^{\alpha-1}
$$

On the other hand, by (A.4) and (A.7), we know that, for $u \in$ $K$ with $\|u\| \geq M_{2}$, there must be

$$
\begin{aligned}
\frac{\left|\left[N_{\infty}(u)\right]^{\prime}(t)\right|}{t^{\alpha-1}\|u\|} \leq & \frac{1}{\Gamma(\alpha-1)} \int_{0}^{t} \frac{(t-s)^{\alpha-2}}{t^{\alpha-1}} \frac{\left|f\left(s, u(s), u^{\prime}(s)\right)-a_{\infty}(s) u(s)-b_{\infty}(s) u^{\prime}(s)\right|}{\|u\|} d s \\
\leq & \frac{1}{\Gamma(\alpha-1)} \int_{I_{u} \cap[0, t]} \frac{(t-s)^{\alpha-2}}{t^{\alpha-1}} \frac{\left|f\left(s, u(s), u^{\prime}(s)\right)-a_{\infty}(s) u(s)-b_{\infty}(s) u^{\prime}(s)\right|}{|u(s)|+\left|u^{\prime}(s)\right|} d s \\
& +\frac{1}{\Gamma(\alpha-1)} \int_{J_{u} \cap[0, t]} \frac{(t-s)^{\alpha-2}}{t^{\alpha-1}} \frac{\left|f\left(s, u(s), u^{\prime}(s)\right)-a_{\infty}(s) u(s)-b_{\infty}(s) u^{\prime}(s)\right|}{M_{2}} d s \leq \varepsilon .
\end{aligned}
$$


By (A.9) and (A.10), we know that

$$
\lim _{u \in K,\|u\| \rightarrow \infty} \frac{N_{\infty} u}{\|u\|}=\theta .
$$

Equation (A.11) together with the boundedness of $(I-G)^{-1}$ will lead to

$$
\lim _{u \in K,\|u\| \rightarrow \infty} \frac{(I-G)^{-1} N_{\infty} u}{\|u\|}=\theta .
$$

\section{Conflicts of Interest}

The authors declare that they have no conflicts of interest.

\section{Authors' Contributions}

All authors contributed equally to the writing of this paper. All authors read and approved the final manuscript.

\section{Acknowledgments}

The research is supported by the National Natural Science Foundation of China [11571197 and 11471187] and STPF of University in Shandong Province [no. J17KA161].

\section{References}

[1] Y. Cui, "Uniqueness of solution for boundary value problems for fractional differential equations," Applied Mathematics Letters, vol. 51, pp. 48-54, 2016.

[2] K. Teng, H. Jia, and H. Zhang, "Existence and multiplicity results for fractional differential inclusions with Dirichlet boundary conditions," Applied Mathematics and Computation, vol. 220, pp. 792-801, 2013.

[3] A. A. Kilbas, H. M. Srivastava, and J. J. Trujillo, Theory and Applications of Fractional Differential Equations, vol. 204 of North-Holland Mathematics Studies, Elsevier Science, Amsterdam, The Netherlands, 2006.

[4] Y. Liu, "On piecewise continuous solutions of higher order impulsive fractional differential equations and applications," Applied Mathematics and Computation, vol. 287/288, pp. 38-49, 2016.

[5] S. Heidarkhani, Y. Zhao, G. Caristi, G. A. Afrouzi, and S. Moradi, "Infinitely many solutions for perturbed impulsive fractional differential systems," Applicable Analysis: An International Journal, vol. 96, no. 8, pp. 1401-1424, 2017.

[6] S. Heidarkhani, A. Salari, and G. Caristi, "Infinitely many solutions for impulsive nonlinear fractional boundary value problems," Advances in Difference Equations, vol. 2016, no. 1, article 196, 2016.

[7] S. Heidarkhani, "Multiple solutions for a nonlinear perturbed fractional boundary value problem," Dynamic Systems and Applications, vol. 23, no. 2-3, pp. 317-331, 2014.

[8] B. P. Rynne, "Spectral properties and nodal solutions for second-order, $m$-point, boundary value problems," Nonlinear Analysis. Theory, Methods \& Applications, vol. 67, no. 12, pp. 3318-3327, 2007.

[9] G. Dai and R. Ma, "Unilateral global bifurcation phenomena and nodal solutions for $p$-Laplacian," Journal of Differential Equations, vol. 252, no. 3, pp. 2448-2468, 2012.
[10] G. Dai, H. Wang, and B. Yang, "Global bifurcation and positive solution for a class of fully nonlinear problems," Computers \& Mathematics with Applications, vol. 69, no. 8, pp. 771-776, 2015.

[11] X. Liu and J. Sun, "Asymptotic bifurcation points, and global bifurcation of nonlinear operators and its applications," Nonlinear Analysis. Theory, Methods \& Applications, vol. 75, no. 1, pp. 7-21, 2012.

[12] B. P. Rynne, "Infinitely many solutions of superlinear fourth order boundary value problems," Topological Methods in Nonlinear Analysis, vol. 19, no. 2, pp. 303-312, 2002.

[13] P. H. Rabinowitz, "Some global results for nonlinear eigenvalue problems," Journal of Functional Analysis, vol. 7, no. 3, pp. 487513, 1971.

[14] E. N. Dancer, "On the structure of solutions of non-linear eigenvalue problems," Indiana University Mathematics Journal, vol. 23, pp. 1069-1076, 1974.

[15] E. N. Dancer, "Global solutions branches for positive maps," Archive for Rational Mechanics and Analysis, vol. 55, no. 3, pp. 207-213, 1974.

[16] Y. Guan, Z. Zhao, and X. Lin, "On the existence of positive solutions and negative solutions of singular fractional differential equations via global bifurcation techniques," Boundary Value Problems, vol. 2016, no. 1, article 141, 2016.

[17] E. Zeidler, Nonlinear Functional Analysis and its Applications, I. Fixed-Point Theorems, Springer, New York, NY, USA, 1986.

[18] K. Deimling, Nonlinear Functional Analysis, Springer, Berlin , Germany, 1985. 


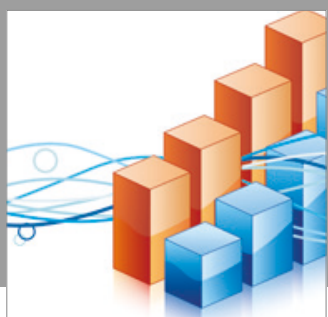

Advances in

Operations Research

vatersals

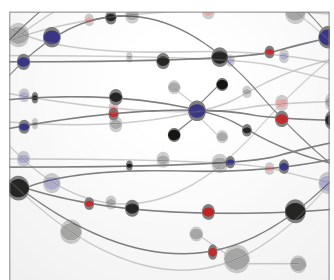

\section{The Scientific} World Journal
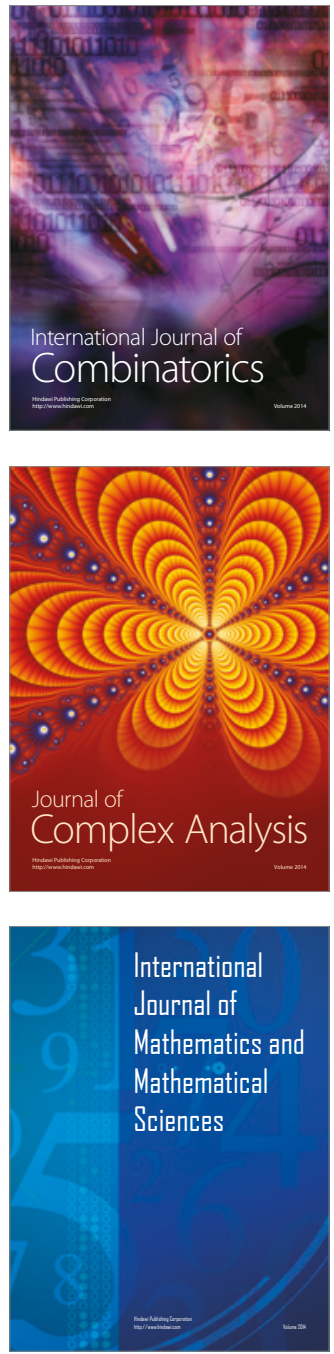
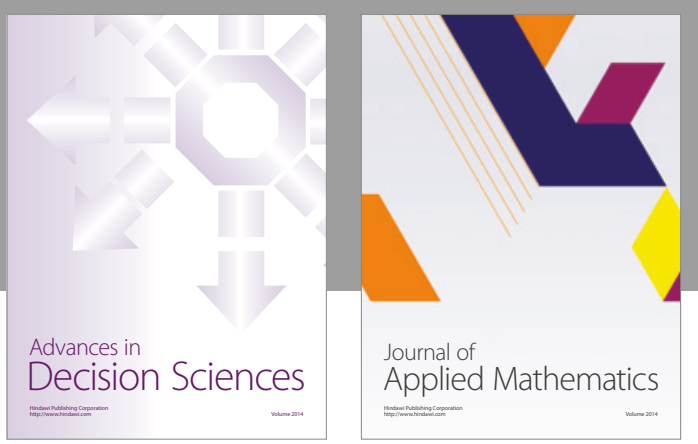

Algebra

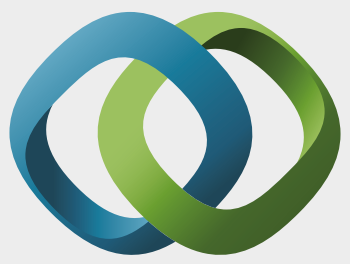

\section{Hindawi}

Submit your manuscripts at

https://www.hindawi.com
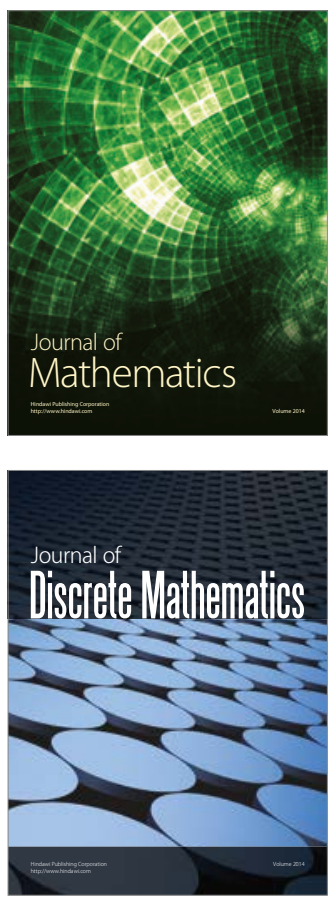

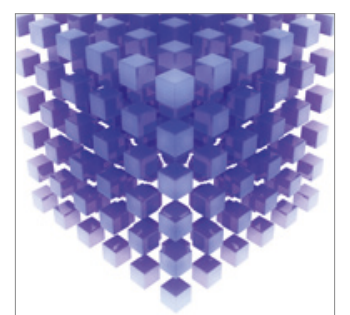

Mathematical Problems in Engineering
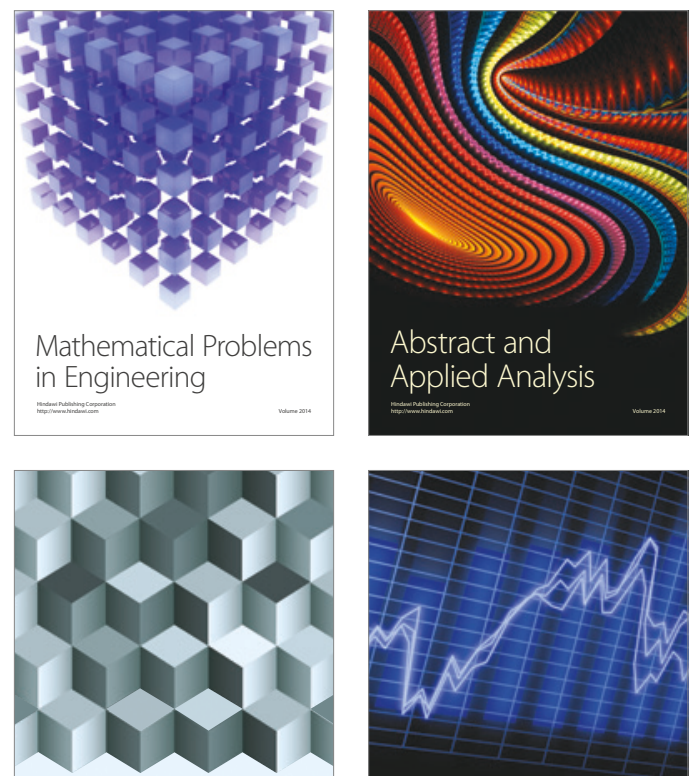

Journal of

Function Spaces

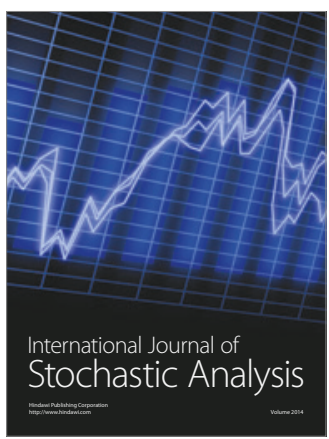

Probability and Statistics
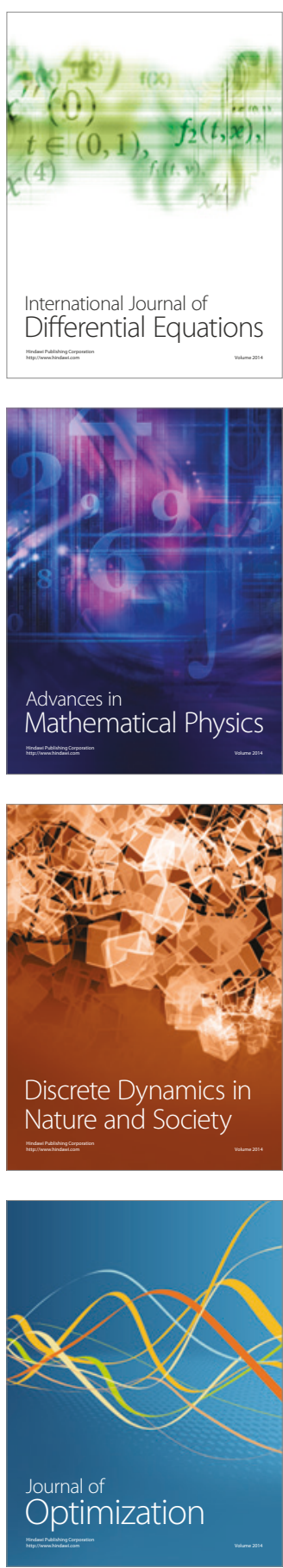